\title{
Elastic and Efficient LiDAR Reconstruction for Large-Scale Exploration Tasks
}

\author{
Yiduo Wang ${ }^{1}$, Nils Funk ${ }^{2}$, Milad Ramezani ${ }^{1}$, Sotiris Papatheodorou ${ }^{2}$, Marija Popović ${ }^{2}$, Marco Camurri ${ }^{1}$, \\ Stefan Leutenegger ${ }^{2}$ and Maurice Fallon ${ }^{1}$
}

\begin{abstract}
We present an efficient, elastic 3D LiDAR reconstruction framework which can reconstruct up to maximum LiDAR ranges $(60 \mathrm{~m})$ at multiple frames per second, thus enabling robot exploration in large-scale environments. Our approach only requires a CPU. We focus on three main challenges of large-scale reconstruction: integration of long-range LiDAR scans at high frequency, the capacity to deform the reconstruction after loop closures are detected, and scalability for longduration exploration. Our system extends upon a state-of-theart efficient RGB-D volumetric reconstruction technique, called supereight, to support LiDAR scans and a newly developed submapping technique to allow for dynamic correction of the 3D reconstruction. We then introduce a novel pose graph clustering and submap fusion feature to make the proposed system more scalable for large environments. We evaluate the performance using two public datasets including outdoor exploration with a handheld device and a drone, and with a mobile robot exploring an underground room network. Experimental results demonstrate that our system can reconstruct at $3 \mathrm{~Hz}$ with $60 \mathrm{~m}$ sensor range and $\sim 5 \mathrm{~cm}$ resolution, while state-of-the-art approaches can only reconstruct to $25 \mathrm{~cm}$ resolution or $20 \mathrm{~m}$ range at the same frequency.
\end{abstract}

\section{INTRODUCTION}

Dense reconstruction is an active research topic. Being able to recover rich geometric information in real time is important for applications such as active mapping [1], [2], obstacle avoidance [3] and industrial inspection [4], [5]. Lower cost and denser LiDAR sensors have come to the market thanks to the focus on self-driving car research. However, large scale exploration and reconstruction still remain challenging problems.

A major challenge in reconstruction is global consistency because the accumulation of some degree of odometry error is unavoidable during large-scale exploration - even for high performance approaches such as LOAM [6]. Error increases with distance travelled and is typically corrected by loop closures in the context of SLAM. These corrections can also be applied to the map representation used within SLAM systems, which are typically point clouds for LiDARbased SLAM. However, point cloud representations lack

This research is supported by the ESPRC ORCA Robotics Hub (EP/R026173/1). M. Fallon is supported by a Royal Society University Research Fellowship and S. Papatheodorou by the President's PhD Scholarship. N. Funk's PhD is funded by SLAMcore Ltd.

1 These authors are with the Oxford Robotics Institute, University of Oxford, UK. \{ywang, milad, mcamurri, mfallon\}erobots.ox.ac.uk

2 These authors are with the Smart Robotics Lab, Department of Computing, Imperial College London, UK \{nils.funk13, s.papatheodorou18, mpopovil, s.leutenegger\}@ic.ac.uk

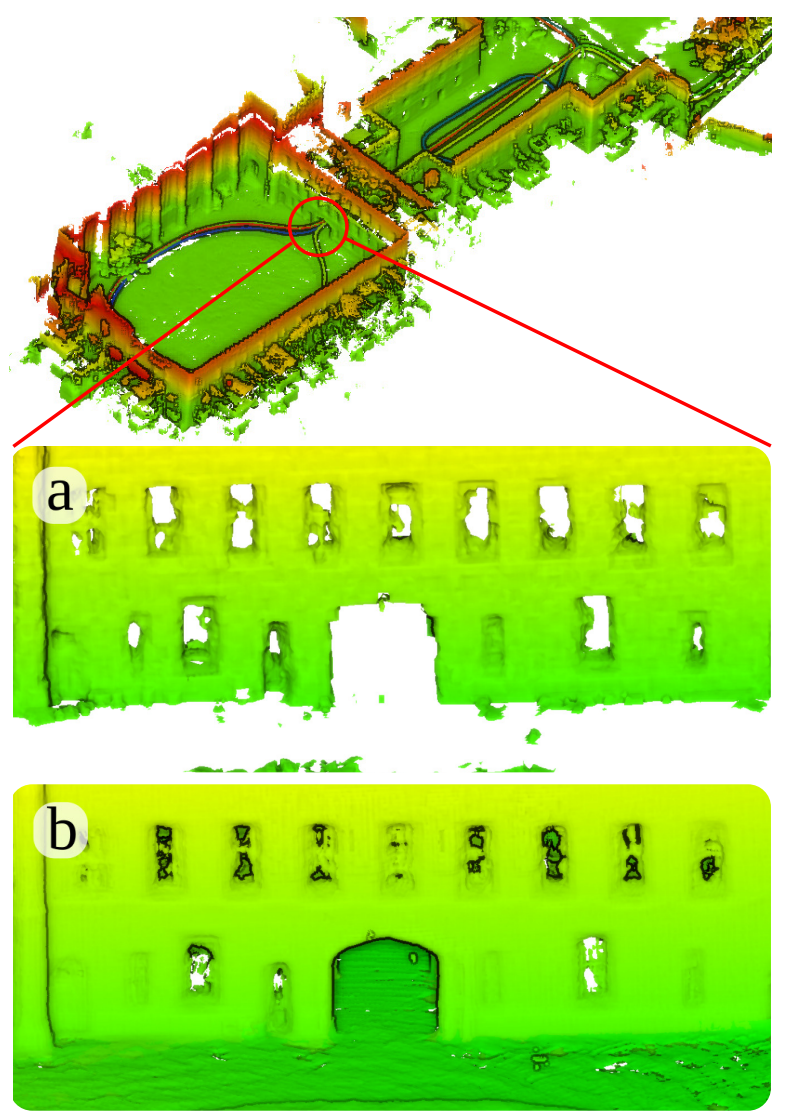

Fig. 1: Exploration trajectory and 3D reconstruction result from our elastic supereight multi-resolution TSDF pipeline on Newer College Dataset. The close-ups focus on the narrow tunnel on the opposite side of the Quad area from experiment's start. (a - the first submap $40 \mathrm{~m}$ away; $\mathrm{b}$ - revisiting after a large loop closure.) More demonstrations are available in the supplementary video: https: //youtu.be/13xz4YnCGW0

the occupancy information necessary for exploration and planning to distinguish between unknown and observedfree voxels. If volumetric or surface mesh reconstructions are built on-the-fly by an exploring robot, the resulting reconstruction would be rigid and impossible to incorporate the effect of loop closures.

Another challenge is finding a good trade-off between the resolution/scale of the reconstruction, and the speed/efficiency of the system. A precise representation of occupancy is important for path planning, especially when navigating through tunnels and door ways such as in Fig. 1 In this work, we greatly expand supereight, a state-of-theart multi-resolution reconstruction framework for RGB-D cameras, to incorporate 3D multi-beam LiDAR scans. It uses 
high resolution in close proximity for path planning and lower resolution at longer ranges for fast reconstruction. We then demonstrate that our approach is more efficient than other state-of-the-art pipelines at high resolutions.

Finally, we also implement a novel system to cluster the SLAM pose graph and reconstruction, inspired by large-scale systems such as the Atlas SLAM framework [7] and SLAM pose graph sparsification techniques [8]-[10]. In our work, we focus on improving the scalability of mapping instead of pose-graph SLAM. Reconstructions of the same physical space are fused together to avoid redundant mapping.

The contributions and features of our research are the following:

- An elastic 3D reconstruction system that supports corrections to its underlying shape, e.g. from loop closures.

- A pose graph clustering and submap fusion strategy that makes the reconstruction's memory usage grow proportionally with the size of the environment rather than the duration of exploration.

- Incorporation of LiDAR into a state-of-the-art reconstruction framework for occupancy mapping, which achieves multi-fps $(3 \mathrm{~Hz})$ full range $(60 \mathrm{~m})$ LiDAR scan integration with high resolution $(\sim 5 \mathrm{~cm})$ to enable high precision motion planning and long range autonomy. To the best of our knowledge, this is the first system that achieves this level of performance.

- Evaluation of the system using real-world datasets in large-scale environments against state-of-the-art methods such as Octomap and Voxgraph.

The remainder of this paper is organised as follows. In Section $[$ we discuss the related work. Section $\amalg$ focuses on the reconstruction method and Section IV] explains how we achieve elasticity. Experimental results are presented in Section V Section VI discusses conclusions and future work.

\section{RELATED WORK}

Dense SLAM and mapping systems are very active areas of research. Our proposed system mainly focuses on two aspects, namely large-scale dense reconstruction, and submaps and elasticity. In this section, we will give a brief review of the most relevant systems.

a) Dense Reconstruction: There is a variety of representations for 3D environments. KinectFusion [11] and ElasticFusion [12] used Truncated Signed Distance Function (TSDF) and surfel respectively to achieve highly detailed reconstructions, utilising GPU to integrate inputs from RGB$\mathrm{D}$ cameras. These sensors typically have a myopic sensing range of only $3 \mathrm{~m}$ as compared to as much as $100 \mathrm{~m}$ for terrestrial LiDAR. Recent work by Park et al. [13] revised the dense surfel model for dense LiDAR SLAM.

Another technique to improve reconstruction scalability is dynamic allocation via data structures such as octrees [14][16] and hash-tables [17], [18]. For instance, Niessner et al. [19] employed Hashing Voxel Grid (HVG) to exploit environment sparsity for large-scale RGB-D mapping using TSDF, which was also incorporated in systems such as BOR $^{2} \mathrm{G}$ [20] and InfiniTAM [21], [22].
Systems designed for navigation and motion planning prefer to use occupancy grids for dense reconstruction to explicitly represent free and unknown space [14]-[16], [23]. Vespa et al. [24] presented supereight, a volumetric SLAM system for RGB-D measurements that uses an efficient octree structure to store either TSDF or Occupancy information at various levels of detail depending on the distance to surfaces. Compared to InfiniTAM [21], supereight demonstrated better efficiency. In this work, we extend supereight to support the much longer ranges from 3D LiDAR sensors, while also utilising the adaptive resolution feature.

b) Submaps and Elasticity: Odometry error accumulated through incremental tracking methods can lead to distortion in the map. Dense SLAM systems such as KineticFusion [11] and ElasticFusion [12] tightly couple their reconstructions with the SLAM trajectory and correct individual past scans to bend the mapped reconstruction upon loop closure. This method, however, suffers from poor scalability in large-scale online operations.

Another technique to improve global consistency is to represent the full reconstruction as a collection of submaps with bounded size. This technique originated in SLAM research such as the Atlas framework by Bosse et al. [7] and DenseSLAM by Nieto et al. [25]. Ho et al. [14] and Reijgwart et al. [26] both exploited submaps to achieve elasticity in dense reconstruction. Their reconstructions are based on OctoMap [27] and Voxblox [3], respectively. Upon loop closure, submaps in both systems can be moved around to keep global consistency.

Our approach is most directly motivated by the work of Reijgwart et al. [26]. Voxgraph incorporates both LiDAR and RGB-D measurements, and was demonstrated to be lightweight enough to run on a drone. The main drawback of this system is the dense data structure of hash-tables. TSDF integration and ESDF computation are all carried out at the finest resolution. The typical parameter setup the authors used for a LiDAR exploration task was short range $(16 \mathrm{~m})$ with coarse resolution $(20 \mathrm{~cm})$. With such parameters, it would be impossible to plan at full LiDAR ranges.

\section{LARGE-SCALE SUPEREIGHT}

In this section, we describe the core reconstruction component of our system, supereight [24], and the significant improvements needed to support long range LiDAR sensing. Supereight is a volumetric, octree-based SLAM pipeline with adaptive resolution that uses Morton codes to achieve efficient spatial octree traversals. Instead of individual voxels, supereight stores blocks which aggregate $8 \times 8 \times 8$ voxels as the finest leaves of the tree. This results in fewer memory allocations and improved cache locality during updates, improving performance. It is also capable of integrating data at different octree levels, further increasing efficiency.

In this work, the mapping component of supereight is connected to a LiDAR SLAM system [28]. We expand both supereight's multi-resolution TSDF (MultiresTSDF) [24] and multi-resolution occupancy (MultiresOFusion) [29] pipelines to incorporate LiDAR inputs. The original RGB-D supereight 

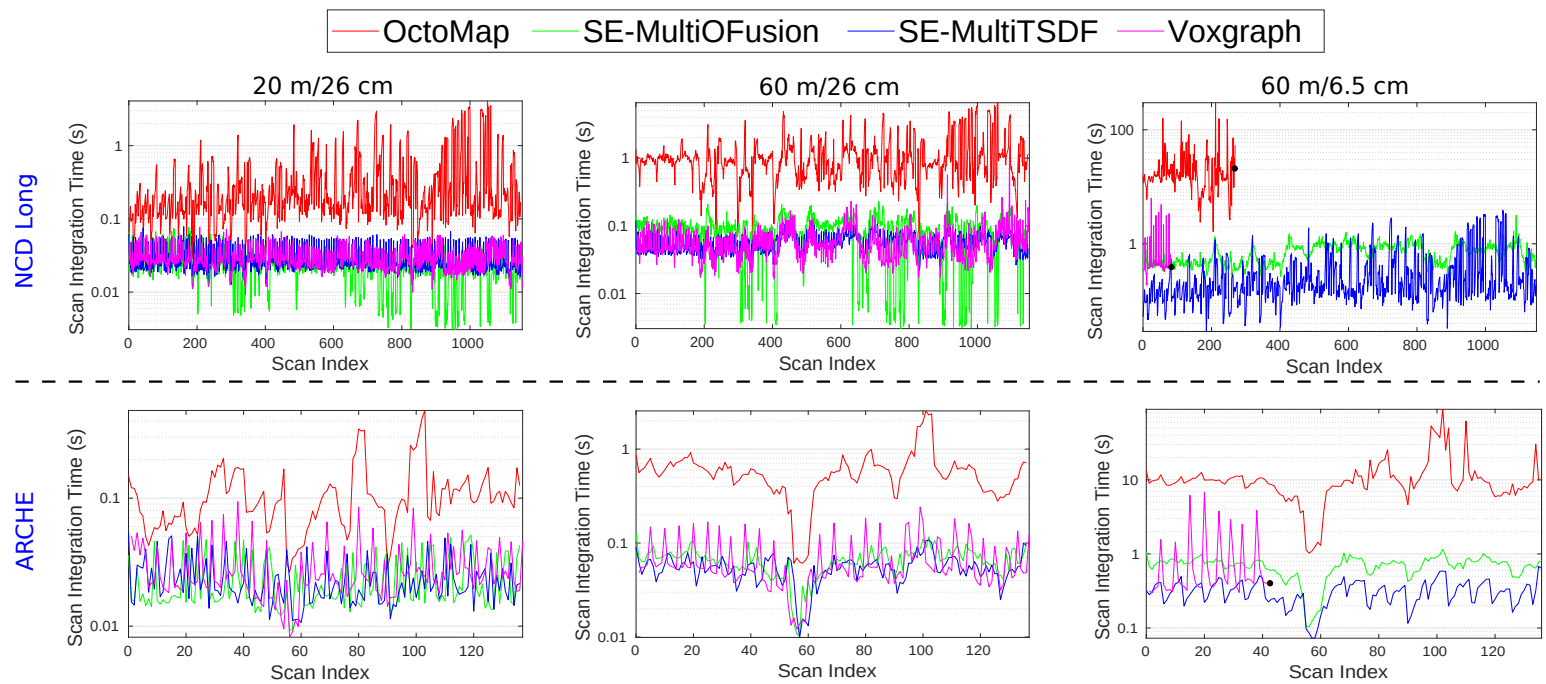

Fig. 2: Integration time per LiDAR scan of different reconstruction systems in large-scale exploration experiments. Our goal is to achieve high resolution at maximum sensor range (right column).

uses a pinhole camera model. To incorporate LiDAR data into the framework, organised LiDAR point clouds are converted to depth images, and the projection model is approximated with a spherical camera model by defining a pair of azimuth and elevation angles for each pixel in the depth image based on sensor specification. Compared to the pinhole camera model, the LiDAR model incorporates a longer range and larger Field of View (FoV), but the distance measurements are sparser. We also create local submaps in this system to replace the single global map in the original pipeline. This is further explained in Sec. IV.

\section{A. Multi-resolution}

Long range measurements from a LiDAR cover a much larger amount of free space than an RGB-D camera. Supereight can update the octree at various levels depending on the effective resolution of the sensor, by updating cubes consisting of several voxels instead of individual voxels. The benefit of this approach is a reduction in the number of octree updates, resulting in reduced integration time [24]. This performance increase is especially important in the case of LiDAR sensors where a single scan may contain measurements ranging from a few meters to $60 \mathrm{~m}$ away.

Due to a larger FoV, it is more likely for LiDAR rays to hit surfaces at shallow angles than those of RGB-D cameras, which results in aliasing artefacts. In this work, we update supereight's integration level selection method for a particular depth measurement to make it suitable for LiDAR sensors. This update reduces aliasing artefacts, increases speed and decreases memory consumption at large distances. For each ray $\mathbf{r}$, we consider the minimum angle between two adjacent LiDAR scan rays, which in turn defines a circular cone. We then determine the scale of update volume at a distance measurement $d_{r}$ the largest block of voxels that fits inside this cone, up to $8 \times 8 \times 8$ voxels. Thus, measurements can be integrated into volumes at adaptively selected resolutions.

We use the propagation strategies described in [24] and [29] for MultiresTSDF and MultiresOFusion, respectively, to keep the hierarchy consistent between different integration levels. Additionally, in MultiresOFusion the maximum occupancy and observed state at the finest integration level are up-propagated to each parent level to provide fast occupancy queries at different levels. MultiresOFusion also explicitly keeps track of free space at the coarsest possible scale while preserving details about unknown space.

\section{B. LiDAR Integration}

LiDAR integration refers to the process of creating a new reconstruction or updating an existing one based on a new scan. The LiDAR used in our experiments is an Ouster OS164. It produces organised dense point clouds of $64 \times 1024$ points at $10 \mathrm{~Hz}$ (i.e. $655 \mathrm{k}$ points/s), with a vertical FoV of $33.2^{\circ}$ and a horizontal FoV of $360^{\circ}$. Scans are converted from point clouds to spherical range images to facilitate their inclusion into supereight.

The MultiresOFusion pipeline stores the occupancy probability in log-odds form which results in free, unknown and occupied voxels having negative, zero and positive log-odds values, respectively. Occupancy update follows the convention of adding a new log-odds measurement [27], [30]. The log-odds measurement along a ray is a distance-dependent piecewise linear function explained in detail in [29].

The integration process for the MultiresTSDF pipeline is described in [24]. To avoid artefacts when using long-range LiDAR scans, we modify the pipeline so that the TSDF truncation bound adapts to the integration level.

\section{Runtime Performance}

To evaluate our system's efficiency when integrating LiDAR scans, we tested it using the Newer College Dataset (NCD) [31] and the dataset made available with [26] (ARCHE). NCD consists of two experiments with different durations, and we present in this paper the 44 min one (NCD Long). Both datasets were of large-scale (approximately $135 \times 225 \mathrm{~m}^{2}$ for NCD and $70 \times 160 \mathrm{~m}^{2}$ for ARCHE) with 

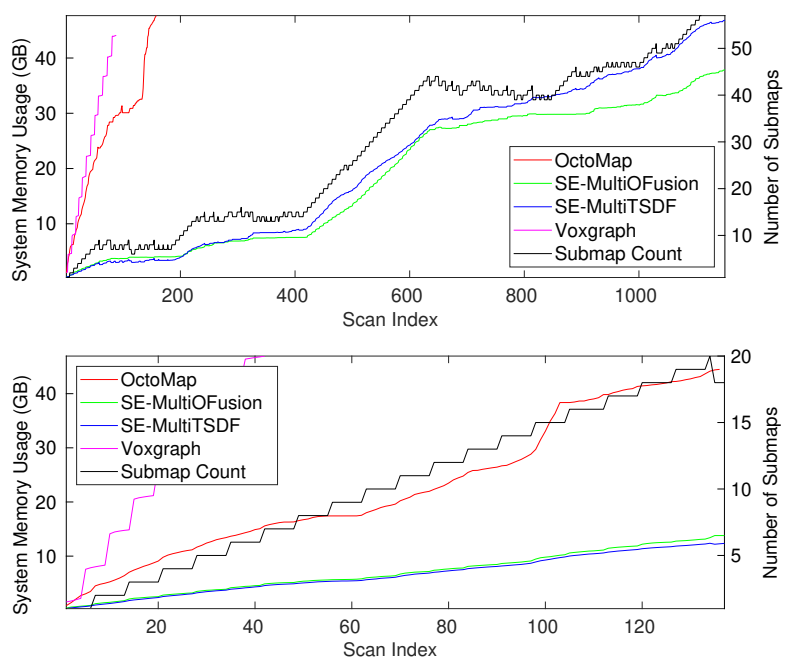

Fig. 3: Memory usage of each pipeline in the NCD Long (top) and ARCHE (bottom) experiments with $60 \mathrm{~m}$ range and $6.5 \mathrm{~cm}$ resolution. Memory usage of our pipelines had a non-linear profile in NCD Long because of the submap fusion feature (Sec. IV-C).

an Ouster OS1-64 LiDAR and a RealSense camera (D435i for NCD and D415 for ARCHE).

The baseline algorithms we chose for comparison are OctoMap [27] and Voxgraph [26], to assess the respective Occupancy and TSDF pipelines. For these experiments, we fed one point cloud every $2 \mathrm{~m}$ travelled into the reconstruction systems. All reconstruction computations were performed on a laptop with an Intel $^{\circledR}$ Xeon E3-1505M v6 CPU, 16 GB of RAM and 32 GB of swap memory.

We evaluated the computation time using three different sets of maximum scan range and voxel resolutions:

- $20 \mathrm{~m}$ max range with $26 \mathrm{~cm}$ resolution

- $60 \mathrm{~m}$ max range with $26 \mathrm{~cm}$ resolution

- $60 \mathrm{~m}$ max range with $6.5 \mathrm{~cm}$ resolution

Fig. 2 shows the integration time at the different range/resolution combinations for NCD Long and ARCHE experiments (top and bottom rows, respectively). We focus on mapping at high resolution $(6.5 \mathrm{~cm})$ with maximum LiDAR range $(60 \mathrm{~m})$, which is presented in the right column of Fig. 2. In both experiments, Voxgraph terminated early due to memory limits, as did OctoMap in NCD Long. Fig. 3 shows the memory consumption of each pipeline, as well as the growth of submaps in the proposed system. The memory usage of OctoMap and Voxgraph increases more quickly than both supereight pipelines, thus illustrating how supereight's multi-resolution improves the memory efficiency of the reconstruction, allowing it to scale to larger environments.

Overall, OctoMap is the least efficient of the evaluated methods. With coarse resolutions, Voxgraph exhibits similar performance to supereight. However, at $6.5 \mathrm{~cm}$ resolution, the MultiresTSDF pipeline is faster than Voxgraph, while MultiresOFusion is on a par with Voxgraph.

\section{Submaps AND Elasticity}

In this section, we detail the components of the elastic reconstruction pipeline, which is shown in Fig. 4

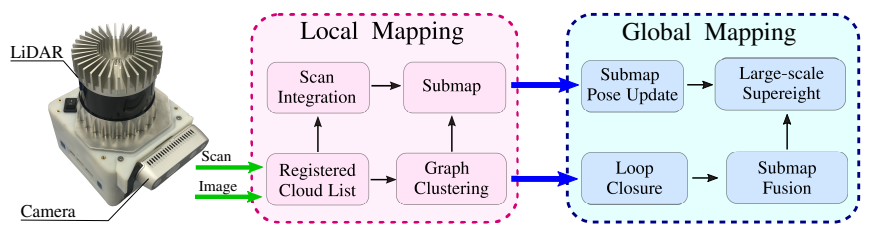

Fig. 4: An overview of the system. Large-scale Supereight is the dense reconstruction core described in Sec. III] and all other components are specially designed for the proposed system. Registered Cloud List is the input. Local mapping clusters the SLAM pose graph and integrates individual LiDAR scans into submaps. Global mapping updates poses of submaps upon loop closures and fuses overlapping submaps together. See Sec. IV for more details.

Our pose graph SLAM system [28] fuses the sensors signals described in Sec. III-C to compute relative odometry which is locally consistent with drift rates in the order of $1 \mathrm{~m}$ per $100 \mathrm{~m}$ travelled. In this way we collect a sequence of point clouds which are registered to one another locally, as well as a corresponding relative pose estimate for the robot/device. Upon loop closures, we form a full pose graph. We call this a Registered Cloud List. The pose graph SLAM system is external to our mapping pipelines, and systems such as [6], [32] could provide be more advanced options.

In our frame convention, the Map frame $\{\mathcal{M}\}$ defines a global fixed frame of reference. The base frame of the device at time $k$ is defined as $\left\{\mathcal{B}_{k}\right\}$. The SLAM system provides a pose graph with $Q+1$ nodes $X_{k}, k \in\{0, \ldots, Q\}$. Each node describes the estimated pose of the device expressed in the map frame ${ }^{\mathcal{M}} \mathbf{T}_{\mathcal{B}_{k}} \in \mathbf{S E}(3)$. The graph's topology consists of both odometry edges (i.e. connecting two consecutive nodes) and loop closure edges.

Each node of the graph is associated with a raw point cloud from the LiDAR. The point clouds have a fixed number of points $p \in \mathbb{R}^{3}$ expressed in the LiDAR frame $\{\mathcal{L}\}$. Given a node $X_{k}$ and its associated point cloud $C_{k}$, the pose of the LiDAR ${ }^{\mathcal{M}} \mathbf{T}_{\mathcal{L}_{k}}$ can be computed as ${ }^{\mathcal{M}} \mathbf{T}_{\mathcal{L}_{k}}={ }^{\mathcal{M}} \mathbf{T}_{\mathcal{B}_{k}}{ }^{\mathcal{B}} \mathbf{T}_{\mathcal{L}}$, where ${ }^{\mathcal{B}} \mathbf{T}_{\mathcal{L}} \in \mathbf{S E}(3)$ is a static transform known by design.

The output of the reconstruction system consists of $N+1$ submaps. Each submap $\mathcal{S}_{i}, i \in\{0 \ldots N\}$ contains:

- The reconstruction as an occupancy map or TSDF

- The root pose of $\mathcal{S}_{i}$

- The node indices and scans used to construct $\mathcal{S}_{i}$

A submap's root pose defines the submap's transformation with respect to the map frame ${ }^{\mathcal{M}} \mathbf{T}_{\mathcal{S}_{i}}$.

\section{A. Graph Clustering}

The graph clustering module processes the pose graph of the Registered Cloud List and groups graph nodes together into different submaps. The clustered graph further guides scan integration (Sec. IV-B and submap fusion (Sec. IV-C).

To perform clustering, we first divide the pose graph edges into odometry and loop closure edges. Odometry edges represent constraints between consecutive pairs of nodes, while loop closure edges are the constraints between nodes that are distant in the graph but correspond to similar scans of revisited places.

If there are no loop closures, grouping into submaps is based only on the odometry chain with a distance $\lambda_{\text {odom }}$. 


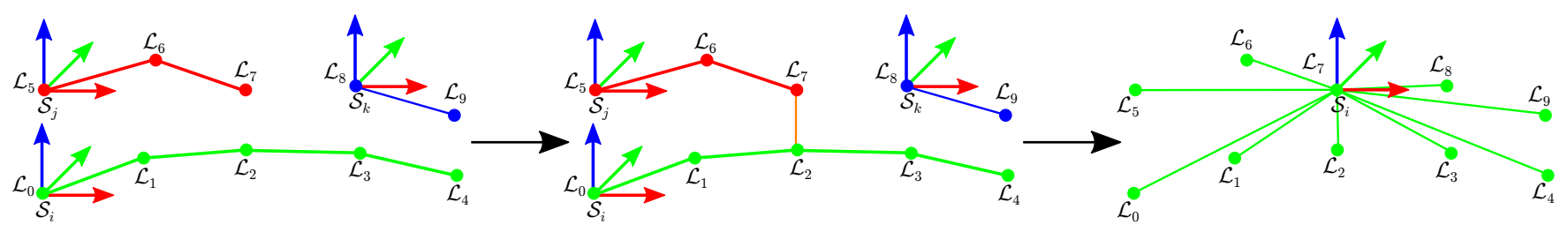

Fig. 5: An example of graph clustering and submap fusion based around a loop closure. Nodes $\mathcal{L}_{0: 4}$ and $\mathcal{L}_{5: 9}$ represent the two traversals of a location from a pose graph. $\mathcal{L}_{0: 4}$ belong to green submap $\mathcal{S}_{i}, \mathcal{L}_{5: 7}$ to red submap $\mathcal{S}_{j}$ and $\mathcal{L}_{8: 9}$ to blue submap $\mathcal{S}_{k}$. Because these nodes have been grouped together by the graph clustering (Sec. IV-A), these three submaps are all merged into submap $\mathcal{S}_{i}$.

In this case, the first node $X_{i, 0}$ of submap $\mathcal{S}_{i}$ defines the submap's root pose ${ }^{\mathcal{M}} \mathbf{T}_{\mathcal{S}_{i}}$ with its corresponding LiDAR pose ${ }^{\mathcal{M}} \mathbf{T}_{\mathcal{L}_{i, 0}}$.

For the subsequent nodes, we compute the distance travelled along the pose graph from the root pose. If a new node is within the distance threshold $\lambda_{\text {odom }}$, the associated LiDAR scan is integrated into $\mathcal{S}_{i}$ according to Sec. IV-B When the new node exceeds the distance threshold, a new submap $\mathcal{S}_{i+1}$ is spawned with that node. This is based on the assumption that the odometry drift is proportional to distance travelled.

Upon loop closure, we cluster together nodes that are within a threshold $\lambda_{\text {cluster }}$ around the pair of nodes that form the closure. Fig. 5 presents an example of clustering. In this example, $\mathcal{L}_{2}$ and $\mathcal{L}_{7}$ are connected by a loop closure edge. We then compute the distances from every surrounding node to this loop closure pair along the pose graph, again assuming that odometry drift is proportional to distance travelled. In the case of $\mathcal{L}_{9}$, its distance is computed as:

$$
\begin{aligned}
& d_{\mathcal{L}_{7}, \mathcal{L}_{9}}=d_{\mathcal{L}_{7}, \mathcal{L}_{8}}+d_{\mathcal{L}_{8}, \mathcal{L}_{9}} \\
& =\left\|{ }^{\mathcal{M}} \mathbf{t}_{\mathcal{L}_{7}}-{ }^{\mathcal{M}} \mathbf{t}_{\mathcal{L}_{8}}\right\|+\|\|^{\mathcal{M}} \mathbf{t}_{\mathcal{L}_{8}}-{ }^{\mathcal{M}} \mathbf{t}_{\mathcal{L}_{9}} \|
\end{aligned}
$$

and because $d_{\mathcal{L}_{7}, \mathcal{L}_{9}}<\lambda_{\text {cluster }}, \mathcal{L}_{9}$ is included in this cluster. Loop closure clusters guide submap fusion in Sec. IV-C.

\section{B. Scan Integration}

After clustering, a point cloud $C_{k}$ at node $X_{k}$ is integrated into the submap $\mathcal{S}_{i}$. We first compute the relative pose between $\mathcal{L}_{k}$ and $\mathcal{S}_{i}$ :

$$
{ }^{\mathcal{S}_{i}} \mathbf{T}_{\mathcal{L}_{k}}={ }^{\mathcal{M}} \mathbf{T}_{\mathcal{S}_{i}}^{-1} \mathcal{M}_{\mathbf{T}_{\mathcal{B}_{k}}}{ }^{\mathcal{B}} \mathbf{T}_{\mathcal{L}}
$$

When $X_{k}$ is the first node of a submap, its cloud $C_{k}$ creates the initial reconstruction of $\mathcal{S}_{i}$ and ${ }^{\mathcal{M}} \mathbf{T}_{\mathcal{S}_{i}}={ }^{\mathcal{M}} \mathbf{T}_{\mathcal{L}_{k}}$.

Using ${ }^{\mathcal{S}_{i}} \mathbf{T}_{\mathcal{L}_{k}}$, we transform every point $p \in C_{k}$ from the LiDAR frame $\left\{\mathcal{L}_{k}\right\}$ to the submap frame $\left\{\mathcal{S}_{i}\right\}$. We then update the submap reconstruction according to Sec. III-B

\section{Submap Fusion}

Submap fusion merges the submaps where a loop closure is detected. For each loop closure cluster described in Sec. IVA we search through all existing submaps and find those that contain nodes from this cluster. These submaps are then fused together as illustrated in Fig. 5

To fuse the submaps $\mathcal{S}_{j}$ and $\mathcal{S}_{i}$, we first need to transform every voxel of $\mathcal{S}_{j}$ with coordinates $v_{j} \in \mathbb{R}^{3}$ into the coordinate system of $\mathcal{S}_{i}$ to obtain $v_{i}$ :

$$
\left[\begin{array}{c}
v_{i} \\
1
\end{array}\right]={ }^{\mathcal{S}_{i}} \mathbf{T}_{\mathcal{S}_{j}}\left[\begin{array}{c}
v_{j} \\
1
\end{array}\right],{ }^{\mathcal{S}_{i}} \mathbf{T}_{\mathcal{S}_{j}}={ }^{\mathcal{M}} \mathbf{T}_{\mathcal{S}_{i}}^{-1}{ }^{\mathcal{M}} \mathbf{T}_{\mathcal{S}_{j}}
$$

If the voxel $v_{i}$ falls out of the current scanned space in $\mathcal{S}_{i}$, it will be newly allocated and assigned as $v_{j}$ in $\mathcal{S}_{j}$. Otherwise, the voxel data in $v_{j}$ will be integrated into $v_{i}$ following the model in Sec. III-B.

Submap fusion prevents new submaps from being spawned when the same space is revisited. Updating an existing submap is more memory efficient than creating two overlapping submaps. This has the advantage of making the reconstruction complexity grow proportionally with the amount of space explored rather than the duration of the exploration.

The memory usage of the long range $(60 \mathrm{~m})$ high resolution $(6.5 \mathrm{~cm})$ NCD Long experiment, as presented in Fig. 3 . demonstrates such benefit. Fig. 3 also presents the growth of submaps in the experiment. Up to scan 400 in the NCD Long experiment, the number of submaps has limited growth because the experiment stays within the Quad area and loops are closed. There after the device explored the wide open Parkland area - with submap growth becoming linear. Ideally submap growth should have fully plateaued when revisiting the same area regularly (after scan 600). Improving submap reduction to achieve the plateauing is a future work.

\section{Submap Pose Update}

The submap pose update module ensures global consistency in the reconstruction. When loop closure occurs, the pose graph and poses of the SLAM system are updated.

The naive approach of updating all the submaps upon loop closure is computationally infeasible for real-time applications, as discussed by Sodhi et al. [15]. Instead, we define a criterion to determine whether a submap $\mathcal{S}_{i}$ needs to be corrected, such that a large-scale reconstruction can be selectively and efficiently updated. Let $\mathcal{M} \hat{\mathbf{T}}_{\mathcal{S}_{i}}$ denote the updated transformation ${ }^{\mathcal{M}} \mathbf{T}_{\mathcal{S}_{i}}$ of $\mathcal{S}_{i}$ with respect to the map frame $\mathcal{M}$. We empirically determined translational and rotational thresholds which trigger a submap correction, respectively $10 \mathrm{~cm}$ and $2.5^{\circ}$. If the position/rotation change exceeds its threshold, the submap is corrected:

$$
\left\|{ }^{\mathcal{M}} \hat{\mathbf{t}}_{\mathcal{S}_{i}}-{ }^{\mathcal{M}} \mathbf{t}_{\mathcal{S}_{i}}\right\|>\lambda_{\text {update }} \vee\left\|^{\mathcal{M}} \hat{\mathbf{R}}_{\mathcal{S}_{i}}^{-1 \mathcal{M}} \mathbf{R}_{\mathcal{S}_{i}}\right\|>\theta_{\text {update }}
$$

Because we do not maintain a global map in our pipelines, this update need only correct the root poses of the submaps, with no additional global map fusion required.

\section{EXPERIMENTS AND EVALUATION}

Sec. III-C presents the computation time and memory usage of the proposed system compared to OctoMap and Voxgraph. In this section, we evaluate the global consistency of the proposed online elastic reconstruction pipeline 


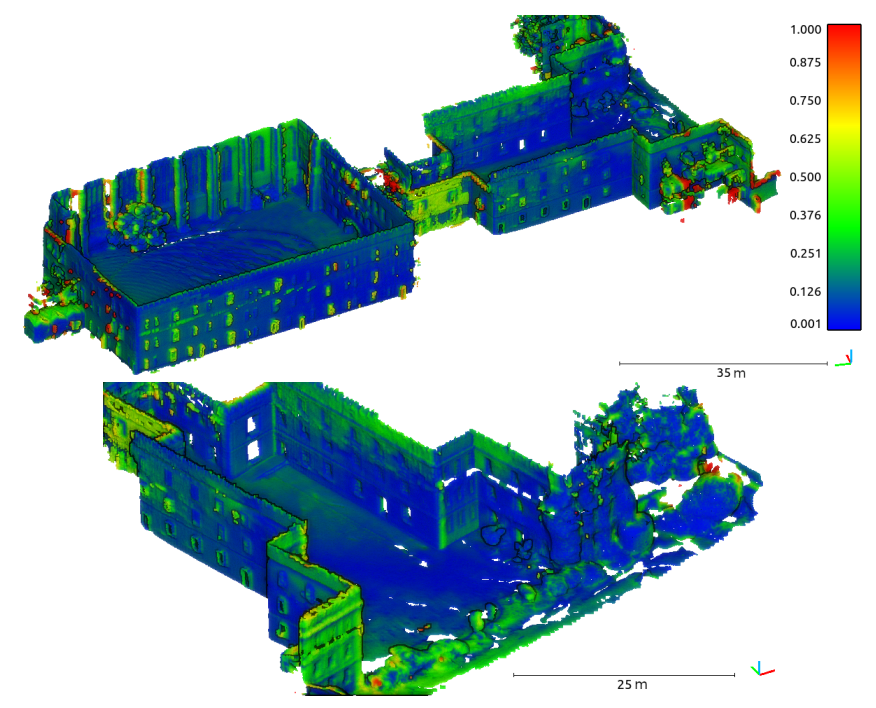

Fig. 6: Evaluation of reconstruction accuracy using point-to-point distance compared against ground truth.

using MultiresTSDF and test the path planning application of MultiresOFusion. To assess the level of global consistency achieved via submap elasticity, we compared the MultiresTSDF map with the ground truth of NCD.

For path planning, we tested on a dataset of a mobile robot exploring an network of rooms in a underground mine, and used the MultireOFusion pipeline to create a high resolution occupancy map so as to test suitability for path planning.

\section{A. Reconstruction Accuracy}

In Fig. 6, we present the result of the MultiresTSDF reconstruction on the NCD Long experiment [31]. It is an exploration task spanning nearly $2.2 \mathrm{~km}$ in a large-scale college campus. Given the scale, the $60 \mathrm{~m}$ maximum sensor range and $6.5 \mathrm{~cm}$ voxel resolution is needed for this reconstruction.

To evaluate the map quality, we fused all the submaps generated at the end of the experiment into one global MultiresTSDF reconstruction and extracted all the vertices of the mesh to form a full point cloud of the explored environment. We then compared the extracted cloud with a ground truth map collected with a Leica BLK 360, a survey grade tripod-based laser scanner. The ground truth cloud was downsampled to $5 \mathrm{~cm}$ to match the resolution of our reconstruction. We used CloudCompar ${ }^{1}$ to align the ground truth and the reconstructed point cloud and to compute the point-to-point distance error between them.

For about $90 \%$ of the points, the distance error is less than $50 \mathrm{~cm}$. The tunnel and the doorway connecting each section of the campus were reconstructed with high accuracy. This is demonstrated in detail in Fig. 11, where the finely detailed mesh of the tunnel enables tasks such as robot path planning. The reconstruction results of the ARCHE experiment are presented in the supplementary video.

We note that the Ouster LiDAR used in the experiments is not as accurate as devices such as Velodyne, but Ouster's wide vertical FoV is valuable for our intended usecase of exploration and planning rather than mapping specifically.

\footnotetext{
${ }^{1}$ https://www.danielgm.net/cc/
}

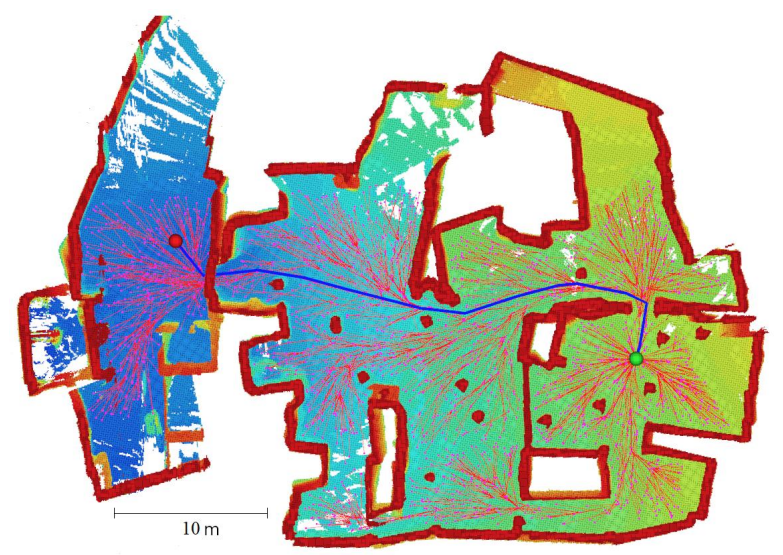

Fig. 7: Using the reconstruction result for path planning in an underground room network. Green sphere - start; red sphere - end; red tree with magenta nodes - RRT*; blue trajectory - planned path.

\section{B. Path Planning in Underground Network}

To test the MultiresOFusion pipeline on a realistic path planning application, we collected a dataset in an underground mine consisting of a room network hewn from the rock. The dataset was collected with the same sensor platform as in NCD, but mounted on a Husky wheeled robot. We ran the pipeline with $6.5 \mathrm{~cm}$ resolution and $60 \mathrm{~m}$ range again. The resultant occupancy map was then used by an RRT* [33] path planner to compute the shortest collision free path between two locations. The result is presented in Fig. 7 . the volumetric reconstruction is highly detailed, giving clear definition even in narrow doorways and corridors. This allowed the path planner to find the optimal path to the goal despite obstacles such a support pillars.

\section{CONCLUSION AND FUtURE WORK}

To summarise, our proposed system can efficiently reconstruct large-scale environments at $3 \mathrm{~Hz}$ with high resolution $(\sim 5 \mathrm{~cm})$ using long range LiDAR scans $(60 \mathrm{~m}$ max range). The core data structure of supereight exploits sparsity of the environment with an adaptive resolution representation and allows for better scalability and efficiency in scan integration as compared to state-of-the-art mapping systems such as Voxgraph and OctoMap. Thanks to high resolution reconstruction around obstacles, our mapping result is suitable for navigation and path planning even in challenging situations.

We use submaps to introduce elasticity into the volumetric map. This allows the reconstruction to be corrected with SLAM loop closures during long exploration tasks. Our results demonstrate global consistency in the map when compared to the ground truth. Graph clustering and submap fusion also further improve system's scalability.

To further improve the reconstruction accuracy, we plan to extend supereight to incorporate an undistorted spherical model to account for LiDAR motion distortion and enable high speed operation. In addition, we aim to improve the submap fusion by applying a graph sparsification heuristic based on information theory, similar to [8]-[10], so that the number of maps plateaus when a space is fully explored. 


\section{REFERENCES}

[1] A. Bircher, M. Kamel, K. Alexis, H. Oleynikova, and R. Siegwart, "Receding horizon path planning for 3D exploration and surface inspection," Autonomous Robots, vol. 42, no. 2, pp. 291-306, 2018.

[2] A. Dai, S. Papatheodorou, N. Funk, D. Tzoumanikas, and S. Leutenegger, "Fast frontier-based information-driven autonomous exploration with an MAV," in IEEE Intl. Conf. on Robotics and Automation (ICRA), Paris, France, June 2020.

[3] H. Oleynikova, Z. Taylor, M. Fehr, R. Siegwart, and J. Nieto, "Voxblox: Incremental 3D Euclidean signed distance fields for onboard MAV planning," in IEEE/RSJ Intl. Conf. on Intelligent Robots and Systems (IROS), 2017.

[4] G. A. Hollinger, B. Englot, F. S. Hover, U. Mitra, and G. S. Sukhatme, "Active planning for underwater inspection and the benefit of adaptivity," Intl. J. of Robotics Research, vol. 32, no. 1, pp. 3-18, 2013.

[5] F. S. Hover, R. M. Eustice, A. Kim, B. Englot, H. Johannsson, M. Kaess, and J. J. Leonard, "Advanced perception, navigation and planning for autonomous in-water ship hull inspection," Intl. J. of Robotics Research, vol. 31, pp. 1445-1464, 2016.

[6] J. Zhang and S. Singh, "Loam: Lidar odometry and mapping in realtime." in Robotics: Science and Systems (RSS), vol. 2, no. 9, 2014.

[7] M. Bosse, P. Newman, J. Leonard, M. Soika, W. Feiten, and S. Teller, "An Atlas framework for scalable mapping," in IEEE Intl. Conf. on Robotics and Automation (ICRA), vol. 2, 2003, pp. 1899-1906 vol.2.

[8] N. Carlevaris-Bianco and R. M. Eustice, "Generic factor-based node marginalization and edge sparsification for pose-graph slam," in IEEE Intl. Conf. on Robotics and Automation (ICRA), 2013, pp. 5748-5755.

[9] M. Mazuran, G. D. Tipaldi, L. Spinello, and W. Burgard, "Nonlinear graph sparsification for slam," in Robotics: Science and Systems (RSS), 2014.

[10] J. Vallvé, J. Solà, and J. Andrade-Cetto, "Graph slam sparsification with populated topologies using factor descent optimization," IEEE Robotics and Automation Letters, vol. 3, no. 2, pp. 1322-1329, 2018.

[11] R. A. Newcombe, S. Izadi, O. Hilliges, D. Molyneaux, D. Kim, A. J. Davison, P. Kohi, J. Shotton, S. Hodges, and A. Fitzgibbon, "KinectFusion: Real-time dense surface mapping and tracking," in IEEE Intl. Symp. on Mixed and Augmented Reality, 2011, pp. 127-136.

[12] T. Whelan, S. Leutenegger, R. Salas-Moreno, B. Glocker, and A. Davison, "ElasticFusion: Dense SLAM without a pose graph," in Robotics: Science and Systems (RSS).

[13] C. Park, P. Moghadam, J. Williams, S. Kim, S. Sridharan, and C. Fookes, "Elasticity meets continuous-time: Map-centric dense 3D LiDAR SLAM," arXiv preprint arXiv:2008.02274, 2020.

[14] B.-j. Ho, P. Sodhi, P. Teixeira, M. Hsiao, T. Kusnur, and M. Kaess, "Virtual occupancy grid map for submap-based pose graph SLAM and planning in 3D environments," IEEE/RSJ Intl. Conf. on Intelligent Robots and Systems (IROS), vol. 1, no. 2, pp. 2175-2182, 2018.

[15] P. Sodhi, B.-J. Ho, and M. Kaess, "Online and consistent occupancy grid mapping for planning in unknown environments," in IEEE/RSJ Intl. Conf. on Intelligent Robots and Systems (IROS), 2019, pp. 78797886.

[16] D. Duberg and P. Jensfelt, "Ufomap: An efficient probabilistic 3d mapping framework that embraces the unknown," 2020.

[17] M. Klingensmith, I. Dryanovski, S. Srinivasa, and J. Xiao, "Chisel: Real time large scale 3D reconstruction onboard a mobile device using spatially hashed signed distance fields," in Robotics: Science and Systems (RSS), vol. 4, 2015, p. 1.
[18] A. Dai, M. Nießner, M. Zollhöfer, S. Izadi, and C. Theobalt, "Bundlefusion: Real-time globally consistent 3D reconstruction using on-thefly surface reintegration," ACM Transactions on Graphics, vol. 36, no. 4, p. 1, 2017

[19] M. Nießner, M. Zollhöfer, S. Izadi, and M. Stamminger, "Real-Time 3D Reconstruction at Scale Using Voxel Hashing," ACM Transactions on Graphics, vol. 32, no. 6, 2013.

[20] M. Tanner, P. Piniés, L. M. Paz, Ştefan Săftescu, A. Bewley, E. Jonasson, and P. Newman, "Large-scale outdoor scene reconstruction and correction with vision," Intl. J. of Robotics Research, 2018.

[21] O. Kähler, V. A. Prisacariu, C. Y. Ren, X. Sun, P. H. S. Torr, and D. W. Murray, "Very High Frame Rate Volumetric Integration of Depth Images on Mobile Device," IEEE Transactions on Visualization and Computer Graphics, vol. 22, no. 11, 2015.

[22] O. Kähler, V. A. Prisacariu, and D. W. Murray, "Real-time large-scale dense $3 \mathrm{~d}$ reconstruction with loop closure," in Eur. Conf. on Computer Vision (ECCV), 2016, pp. 500-516.

[23] D. De Gregorio and L. Di Stefano, "Skimap: An efficient mapping framework for robot navigation," in IEEE Intl. Conf. on Robotics and Automation (ICRA), 2017, pp. 2569-2576.

[24] E. Vespa, N. Funk, P. H. J. Kelly, and S. Leutenegger, "Adaptiveresolution octree-based volumetric SLAM," in Intl. Conf. on 3D Vision, 2019, pp. 654-662.

[25] J. Nieto, J. Guivant, and E. Nebot, "Denseslam: Simultaneous localization and dense mapping," Intl. J. of Robotics Research, vol. 25, no. 8, pp. 711-744, 2006.

[26] V. Reijgwart, A. Millane, H. Oleynikova, R. Siegwart, C. Cadena, and J. Nieto, "Voxgraph: Globally consistent, volumetric mapping using signed distance function submaps," IEEE Robotics and Automation Letters, vol. 5, no. 1, pp. 227-234, 2020.

[27] A. Hornung, K. M. Wurm, M. Bennewitz, C. Stachniss, and W. Burgard, "OctoMap: An efficient probabilistic 3D mapping framework based on octrees," Autonomous Robots, vol. 34, no. 3, pp. 189-206, Apr. 2013.

[28] M. Ramezani, G. Tinchev, E. Iuganov, and M. Fallon, "Online LiDARSLAM for legged robots with robust registration and deep-learned loop closure," in IEEE Intl. Conf. on Robotics and Automation (ICRA), 2020, pp. 4158-4164.

[29] N. Funk, T. Juan, S. Papatheodorou, M. Popović, P. F. Alcantarilla, and S. Leutenegger, "Multi-resolution 3d mapping with explicit free space representationfor fast and accurate mobile robot motion planning," arXiv preprint, 2020

[30] E. Vespa, N. Nikolov, M. Grimm, L. Nardi, P. H. J. Kelly, and S. Leutenegger, "Efficient octree-based volumetric SLAM supporting signed-distance and occupancy mapping," IEEE Robotics and Automation Letters, vol. 3, no. 2, pp. 1144-1151, Apr. 2018.

[31] M. Ramezani, Y. Wang, M. Camurri, D. Wisth, M. Mattamala, and M. Fallon, "The Newer College Dataset: Handheld LiDAR, inertial and vision with ground truth," in IEEE/RSJ Intl. Conf. on Intelligent Robots and Systems (IROS), 2020.

[32] G. Kim and A. Kim, "Scan context: Egocentric spatial descriptor for place recognition within 3d point cloud map," in IEEE/RSJ Intl. Conf. on Intelligent Robots and Systems (IROS), 2018, pp. 4802-4809.

[33] S. Karaman and E. Frazzoli, "Sampling-based algorithms for optimal motion planning," Intl. J. of Robotics Research, vol. 30, no. 7, pp. 846-894, 2011. 